\title{
Propuesta curricular desde un enfoque bimodal y un multimedia informativo para el curso Recursos Didácticos para la Enseñanza del Inglés
}

\author{
Curricular Proposal on a Blended Approach and an Informative Multimedia for Didactic \\ Resources for the Teaching of English Course
}

\section{Proposta curricular desde um enfoque bimodal e um multimídia informativo para o curso Recursos Didáticos para ensinar Inglês}

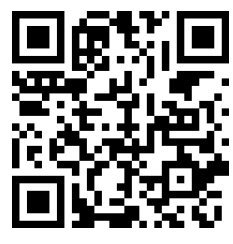

\author{
Silvia Saborío-Taylor \\ Universidad Nacional \\ Heredia, Costa Rica \\ silvia.saborio.taylor@una.cr \\ https://orcid.org/0000-0001-5295-9123
}

Recibido $\cdot$ Received $\cdot$ Recebido: 05 / 03 / 2018
Corregido $\cdot$ Revised $\cdot$ Revisado: 19 / $06 / 2019$
Aceptado $\cdot$ Accepted $\cdot$ Aprovado: 24/ 07/ 2019

Resumen: La investigación se realizó con el objetivo de diseñar una propuesta curricular con un enfoque bimodal para el curso "Recursos Didácticos para la Enseñanza del Inglés" y un multimedia informativo. Se estructuró una metodología en dos etapas: la construcción de una nueva propuesta curricular para impartir el curso bimodal basado en la conceptualización y diseño de recursos tecnológicos; así como la producción de un multimedia informativo para la implementación de recursos didácticos tecnológicos, acorde con la propuesta curricular. Para validar la propuesta y la evaluación del multimedia se llevó a cabo un proceso de investigación evaluativa, por lo que se utilizaron como instrumentos de recolección de datos un análisis de fortalezas, oportunidades, debilidades y amenazas (FODA) (para el estudiantado que recibió el curso) y dos cuestionarios (para docentes en el área de inglés y artes gráficas). Se determinó que la propuesta curricular cuenta con una serie de fortalezas y oportunidades; de igual forma el material multimedia se considera como una herramienta didáctica pertinente para la incorporación de las TIC en la enseñanza del inglés. Por tanto, el proceso de investigación demostró que se logró un efectivo y novedoso diseño de propuesta curricular con un enfoque bimodal y que el multimedia representó un medio de innovación para los recursos didácticos utilizados en el proceso de aprendizaje del inglés.

Palabras claves: Diseño; enfoque bimodal; propuesta curricular; multimedia; TIC. 
doi: http://dx.doi.org/10.15359/ree.23-3.11

URL: http://www.una.ac.cr/educare

CORREO: educare@una.cr

\begin{abstract}
This research conducted in order to design a curricular proposal for the course Didactic Resources for the Teaching of English, based on a blended approach and informative multimedia. The methodology was structured in two stages: the construction of a new curricular proposal to conduct a blended course based on the conceptualization and design of technological resources; also, the production of informative multimedia for the implementation of didactic technological resources according to the curricular proposal. A process of evaluation research was carried out to validate the proposal and evaluate the multimedia; for that reason, a Strengths, Weaknesses, Opportunities and Threats analysis (for students taking the course at the moment) and two questionnaires (for English and Graphic Arts professors) were used for the data collection. The analysis identified that the curricular proposal has a series of strengths and opportunities. Likewise, the multimedia material is considered to be a relevant didactic tool for the inclusion of ICT in English teaching. Therefore, the research process showed the accomplishment of an effective new design for a curricular proposal based on a blended approach; it also showed that the multimedia represented an innovation form for the didactic resources implemented in the English learning process.
\end{abstract}

Keywords: Design; blended approach; curricular proposal; multimedia; ICT.

Resumo: O objetivo dessa investigação foi elaborar uma proposta curricular com um enfoque bimodal para o curso "Recursos Didáticos para ensinar Inglês" e um informativo multimídia. A estruturação de uma metodologia foi feita em duas etapas: a construção de uma nova proposta curricular para dar o curso bimodal baseada na conceituação e disposição dos recursos tecnológicos; a produção de um informativo multimídia para a implementação de recursos didáticos tecnológicos, de acordo com a proposta curricular. Para dar validez a proposta e a avaliação de multimídia, foi necessário um processo de investigação avaliativo, utilizando como instrumentos de recolecção de dados uma análise de fortalezas, oportunidades, debilidades e ameaças (FODA - sigla em espanhol) (para estudantes que receberam o curso) y dois questionários (para docentes na área de inglês e artes gráficas). Ficou determinado que a proposta curricular conta com uma série de fortalezas e oportunidades; de igual forma o material multimídia foi considerado como uma ferramenta didática pertinente para a incorporação das TIC no ensino do inglês. Por isso, o processo de investigação demostrou que foi possível elaborar um novo e eficaz desenho de proposta curricular com um enfoque bimodal, e que a multimídia representou uma forma de inovação para os recursos didáticos utilizados no processo de aprendizagem do inglês.

Palavras-chave: Desenho; enfoque bimodal; proposta curricular; multimídia; TIC.

\title{
Introducción
}

La sociedad actual se ve vinculada en grandes proporciones a un radical desarrollo tecnológico. Es así como las nuevas tecnologías de la información y comunicación (TIC) generan un gran impacto en las diversas actividades de los individuos, sin dejar a un lado la educación.

La tecnología representa hoy un cambio hacia el acceso de información formativa que fundamenta el crecimiento integral de cada persona. Como consecuencia, las TIC han incursionado en los espacios educativos como procesos de innovación que exigen una fundamentación integral sobre cómo proceder en estos cambios. 
Para Cabero (2008), las TIC son representativas dentro la sociedad del conocimiento en el sentido de que influyen en diversas áreas en la forma de la velocidad y volumen de la distribución, la información y en la forma de procesarla. Como consecuencia, las instituciones educativas buscan actualizaciones continuas en los contenidos de los programas, metodologías y recursos con el objetivo de lograr la innovación dentro de la praxis pedagógica. Esta flexibilidad en los programas de estudio podría proporcionar mayor acceso y diversidad en cuanto a formas de aprendizaje, más allá de lo que la educación tradicional podría generar.

Cabero-Almenara y Ruiz-Palmero (2017) agregan que la incorporación de las TIC se debe realizar por medio de una adecuada planificación en los entornos educativos, de forma que se pueda facilitar su inclusión social y digital, lo que conlleva a favorecer el desarrollo de cada persona. De esta forma, se desarrolla como trabajo final de investigación la propuesta curricular con un enfoque bimodal y un multimedia informativo para el curso "Recursos Didácticos para la Enseñanza del Inglés" de la Universidad Nacional; esto, con el fin de dotar de flexibilidad e innovación un programa ya establecido anteriormente, mediante la incorporación planificada de las TIC.

\section{Antecedentes}

Como parte del programa de estudios de la carrera de Bachillerato en la Enseñanza del Inglés del Centro de Investigación y Docencia en Educación (CIDE) de la Universidad Nacional, se ofrece el curso con modalidad presencial "Recursos Didácticos para el Aprendizaje del Inglés" De acuerdo con el programa del curso desarrollada por la Universidad Nacional, Centro de Investigación y Docencia en Educación, División de Educología (2005), este facilitaba la identificación y análisis de los recursos pertinentes al proceso de adquisición del idioma, de acuerdo con los diferentes estilos de aprendizaje, inteligencias múltiples y la diversidad etaria, social y cultural propia de un contexto educativo complejo.

Según planteaba la malla curricular correspondiente para el programa de los cursos de Recursos Didácticos para el Aprendizaje propuesto desde la UNA, CIDE, División de Educología (2005), este consideraba los siguientes ejes temáticos:

- Recursos tecnológicos: televisión, grabadora, laboratorio de idiomas, laboratorio de multimedia, retroproyector, VHS, entre otros.

- Recursos computacionales: paquetes generales y software específico (Word, Power Point, Excel y otros acordes con la labor docente).

- Recursos con desechos.

- Recursos corporales (lenguaje, gestos, teatro, danza, música).

El curso brindaba la posibilidad al estudiantado de investigar, por medio de métodos y técnicas, cómo mejorar la calidad de los recursos didácticos tanto tradicionales como los no tradicionales con el fin de analizar la posibilidad de aplicar estos recursos a la realidad educativa costarricense de secundaria. 
doi: http://dx.doi.org/10.15359/ree.23-3.11

URL: http://www.una.ac.cr/educare

CORREO: educare@una.cr

Como parte de una reestructuración tecnopedagógica, dentro del período de 2008 y 2009, nació el proyecto denominado Implementación de curso Recursos Didácticos para el Aprendizaje bajo un enfoque bimodal para las nueve áreas de la especialidad a las cuales se dirige el curso (Ulate, 2009). El proyecto establecía la implementación de una metodología de igual forma, utilizando para ello el entorno de aprendizaje virtual denominado Moodle. Esta nueva propuesta propuso los mismos ejes temáticos como base para el programa del curso, suponiendo que debía llevar una adaptación para cada disciplina. Los ejes se describen a continuación, según cita el Programa de estudio Recursos Didácticos para el aprendizaje con un enfoque bimodal de la UNA, CIDE, División de Educología (2008):

- Recursos tradicionales: pizarras, carteles, construcción de figuras tridimensionales, televisión-vídeo, radiograbadora, origami, tangrama, retroproyector y recursos bibliográficos.

- Recursos computacionales: CmapTools, Adobe Photoshop, Dreamweaver, ScriptMaster, PhotoWeb, Button Studio, utilización del video bean, utilización de la plataforma de teleformación Moodle.

- Recursos didácticos y su relación con las inteligencias múltiples, los estilos de aprendizaje y de enseñanza, y el planeamiento didáctico.

Como parte de las conclusiones a las cuales llegó el proyecto, según lo expuesto en su informe final respectivo (Ulate, 2009), en cuanto al curso de Inglés, no se logró completar el lo virtual y actualmente se brinda este curso en forma presencial. Además, el análisis del proyecto reflejó una resistencia en menor grado a la aceptación del uso de las TIC. Debido a que el proyecto no logró concretarse satisfactoriamente, se hace necesario diseñar e implementar una propuesta que responda a los requerimientos actuales de la enseñanza del inglés.

Por tanto, se trabajó un trabajo final de investigación, el cual se focalizó en la elaboración de una propuesta curricular renovada para el curso de "Recursos Didácticos para la Enseñanza del Inglés". Se propone un programa que se articulara alrededor de las exigencias de las TIC; con el apoyo de los diferentes recursos tecnológicos disponibles en la Universidad Nacional y en el ciberespacio. Dicha propuesta buscó generar procesos de innovación para el curso, por medio de la propuesta, el diseño y la creación de nuevos multimedia que pudieran beneficiar tanto a estudiantes como a profesorado $y$, desde luego, el proceso de enseñanza y aprendizaje del idioma inglés.

Se formuló una propuesta curricular dirigida específicamente a la enseñanza del idioma inglés que responde al componente pedagógico de la malla curricular de los cursos de Recursos Didácticos para el Aprendizaje (UNA, CIDE, División de Educología, 2005). La propuesta incluyó los diversos enfoques y metodologías para el desarrollo de las diferentes destrezas del habla inglesa, tomando en cuenta que los medios didácticos no perdieran de vista su relación con los estilos de aprendizaje y de enseñanza, inteligencias múltiples y el planeamiento didáctico. 
Además, se incluyó la combinación de estrategias didácticas del modelo de aprendizaje presencial, con las utilizadas en los modelos bimodales de aprendizaje, en el marco de un diseño curricular flexible y actualizado, y como respuesta a sus realidades y necesidades profesionales y académicas que contribuyan al mejoramiento cualitativo de la educación nacional.

Se elaboró, de igual forma, un multimedia como sistema de distribución de información complementario a la propuesta curricular. Con este se informaba y proporcionaba al personal docente sobre nuevas herramientas pedagógicas de aprendizaje significativo que integran la tecnología con los enfoques y metodologías aplicadas a la enseñanza del idioma inglés como lengua extranjera.

\section{Marco teórico}

\section{Enfoque integrado cognitivo-constructivista}

La aplicación de la tecnología en la educación ha sido influenciada por el constructivismo, el cual retoma elementos de una psicología cognitiva. El enfoque constructivista se orienta en el aprendizaje, el estudiantado y el medio ambiente en el cual se desarrolla el aprendizaje. El cognoscitivismo prioriza en los procesos relacionados con el procesamiento de la información, no en la construcción. Según Cabero (2007), cada individuo es un participante activo en la construcción de su entorno, lo que conlleva a la realización de transformaciones internas en su estructura cognitiva. Por su parte, el aprendizaje es un proceso interno que relaciona nueva información con otra ya existente, y esto conduce a que el individuo deba revisar, modificar, reorganizar y diferenciar dicha información, dando paso hacia la concepción constructivista del individuo (Serrano y Pons, 2011).

La idea de una teoría cognoscitivista-constructivista en un ambiente de enseñanzaaprendizaje de idiomas potenciada por tecnología, radica en que el personal docente ya no solo transmite información al estudiantado mientras este último la recibe pasivamente; por el contrario, ahora el sujeto aprendiz interpreta y organiza activamente la información que se le brinda, a la vez que la analiza con el conocimiento previo.

\section{Enfoque comunicativo (CLT, por sus siglas en inglés)}

Nunan (2003) definió el CLT como un enfoque basado en el concepto de que la interacción es la clave para el aprendizaje de un idioma y de que, a su vez, el sujeto aprendiz debe tener oportunidades suficientes para comunicarse en el idioma a aprender durante la lección. Recientemente, la definición del enfoque ha ampliado su alcance, proponiendo que la instrucción de un idioma se basa en experiencias de aprendizaje caracterizadas por logros no lingüísticos, en las cuales se da una conexión entre las actividades que se realizan en clase y las situaciones cotidianas que se presentan fuera del aula. 
doi: http://dx.doi.org/10.15359/ree.23-3.11

URL: http://www.una.ac.cr/educare

CORREO: educare@una.cr

Brown (2001) mencionó una serie de características interconectadas, las cuales describen que en el enfoque CLT el estudiantado debe utilizar el idioma productiva y receptivamente, de forma espontánea en contextos fuera del aula; se da al alumnado la oportunidad de enfocarse en su propio aprendizaje mediante la identificación de su propio estilo de aprendizaje y a través del desarrollo de estrategias apropiadas para lograr un aprendizaje autónomo; el rol del equipo docente pasa a ser facilitador de aprendizaje.

\section{Aprendizaje de idiomas potenciado por tecnología (TELL, por sus siglas en inglés)}

Dudeney y Hockly (2007) definieron TELL como un enfoque dirigido a la enseñanzaaprendizaje de un idioma mediante la utilización de internet y las tecnologías de información y comunicación. Según Brown (2001), se obtienen una serie de beneficios al incluir dichas tecnologías en la instrucción de un idioma, entreellos: práctica multimedia con retroalimentación; individualización en un grupo numeroso; elaboración de tareas o proyectos en parejas o en grupos, ya sea colaborativa o competitivamente; un factor de diversión; aprendizaje exploratorio con gran cantidad de información en el idioma requerido, y un desarrollo de destrezas en ambientes reales.

De esta forma, el enfoque TELL implica el desarrollo del proceso de enseñanza-aprendizaje mediante la utilización de herramientas computacionales que logran, por ejemplo, explorar el mundo por medio de simulaciones; habilitar interfaces que facilitan la creación y utilización de presentaciones y sitios web, que reflejen temas atractivos y significativos; generar una comunicación auténtica con otros aprendices del idioma alrededor del mundo, entre otras.

\section{Taxonomía revisada de Bloom para la era digital}

La taxonomía revisada de Bloom ha sido una herramienta clave para el planeamiento y el desarrollo de los procesos de enseñanza-aprendizaje. Sin embargo, es determinante tomar en cuenta nuevas inclusiones pertinentes a la última actualización a la taxonomía llevada a cabo por Lorin Anderson en los años noventa (Churches, 2009). Si bien la taxonomía de Bloom tiene un fundamento conductista, las nuevas inclusiones atienden a las habilidades, competencias y espectros generados a partir de la inclusión de las TIC en los escenarios educativos. Como consecuencia, la taxonomía ayuda a generar un proceso constructivista, ya que se incluyen habilidades relacionadas con la creación, tales como: programar para crear aplicaciones, filmar videos, producir en espacios digitales, publicar a través de la web, presentar información por medio de herramientas de presentación digitales, entre otras.

La pedagogía y la enseñanza, en la era de la información, están enfocadas en dirigir al estudiantado desde las habilidades del pensamiento de orden inferior (LOTS, por sus siglas en inglés) hacia las habilidades de pensamiento de orden superior (HOTS, por sus siglas en inglés). Cada docente desarrolla, entonces, metodologías que permitan potenciar en el estudiantado 
habilidades como la aplicación, la creación, la elaboración, la innovación, así como el análisis y evaluación de procesos. En ese sentido, la función de la educación se basa en modelar y facilitar en el estudiantado los mecanismos para que logren construir el conocimiento mediante estrategias de colaboración (Cabero, 2001).

\section{Metodología}

\section{Construcción de la propuesta curricular}

Se tomó la iniciativa de construir una nueva propuesta curricular para impartir el curso donde se combine el trabajo presencial con el virtual. Este se basaría en el diseño e implementación de recursos pre-tecnológicos, pero dando mayor énfasis a los recursos tecnológicos aplicados específicamente a los escenarios educativos del habla inglesa.

Se pusieron en práctica los fundamentos pedagógicos y metodológicos para la elaboración de un diseño curricular, partiendo del establecimiento de la finalidad del curso en sí. El diseño se reestructuró a partir de los ejes temáticos presentes en la malla curricular de los cursos de Recursos Didácticos para el Aprendizaje; sin embargo, se reestructuró metodológica y evaluativamente, y se enriquecen los contenidos según la bimodalidad.

Es importante destacar queal ser este un curso bimodal, se trabajó en lograr una integración efectiva con un máximo aprovechamiento de las sesiones presenciales y virtuales de acuerdo con los ejes temáticos. Igualmente, se reformuló el programa de curso de forma que también se tomara en cuenta la comunicación sincrónica y asincrónica, el aprendizaje significativo y el trabajo colaborativo entre las personas participantes. Además, el diseño buscó potenciar el papel protagónico del estudiantado como constructor del conocimiento y el equipo docente como facilitador de información.

Se trabajó en la definición del tratamiento conceptual y la planeación del curso. Para ello, se diseñó un cronograma de trabajo en forma detallada el cual incluía el número de semana, el tema a desarrollar y preguntas generadoras, la estructura de los contenidos, las actividades (tanto presenciales como virtuales) y evaluación de aprendizaje, y por último, los medios y materiales que se utilizan.

Cabe mencionar que dicha propuesta curricular debió tomar en cuenta principios básicos para el diseño de un curso y la metodología para un enfoque bimodal, fundamentos teóricos y prácticos para la enseñanza-aprendizaje del idioma inglés y la integración efectiva de la tecnología en el proceso. Además, este debió ajustarse a los estándares propuestos por la Unidad de Educología (con base en la normativa universitaria) para la elaboración de un programa de curso. Por lo tanto, la propuesta curricular para el curso "Recursos Didácticos para la Enseñanza del Inglés" desde un enfoque bimodal incluye: descripción del curso, problema, preguntas generadoras, ejes temáticos, estrategia metodológica, estrategia de evaluación y cronograma de trabajo. 
doi: http://dx.doi.org/10.15359/ree.23-3.11

URL: http://www.una.ac.cr/educare

CORREO: educare@una.cr

Una vez que se concluyó con el diseño del curso, se presentó a la coordinadora de curso para su revisión, luego fue remitido al Consejo Académico de la División de Educología para su aprobación. Posteriormente, la investigadora impartió el curso en la Sede Interuniversitaria de Alajuela, durante el segundo ciclo del año 2010 con el apoyo de diversos recursos tecnológicos, tales como el portafolio electrónico.

\section{Producción del multimedia}

Se trabajó también en la producción de un multimedia informativo para la implementación de recursos didácticos para la enseñanza del inglés basados en las TIC, acorde con la propuesta curricular y de forma que cumpliera con una metodología didáctica específica en función a la distribución de información de acuerdo con el objetivo general de este y con una población meta previamente establecida.

Además, se definió una metáfora pedagógica atractiva pero acorde con el contenido; un método de navegación no lineal y presencia de diversas zonas de interacción. Conjuntamente, como resultado del guión didáctico, el producto consta de diez módulos dentro de los cuales se incluye información introductoria al multimedia, a la implementación de las TIC en la enseñanza del inglés y, por supuesto, la identificación, creación e implementación de diversas herramientas tecnológicas en los entornos educativos del habla inglesa.

Es importante aclarar que para lograr potenciar una utilización eficiente del multimedia por parte de la población meta, se trabajó también en la producción de un manual de público usuario.

\section{Resultados, análisis y discusión}

Para ejecutar la validación de la propuesta y la evaluación del multimedio se llevó a cabo un proceso de investigación evaluativa. Al respecto, se indica que este tipo de investigación se enfoca en poder "proporcionar información para la planificación del programa, su realización y su desarrollo" (Suchman, 1967, citado por Correa, Puerta y Restrepo,1996, p. 31).

\section{Validación de la propuesta curricular}

Con el propósito de obtener criterios para la toma de decisiones en relación con la estructura, el funcionamiento y el desarrollo de la propuesta curricular que pretendía sustentar el programa del curso "Recursos Didácticos para la Enseñanza del Inglés" se realizó un análisis de fortalezas, oportunidades, debilidades y amenazas (FODA).

El instrumento respondió a la necesidad de validar la aplicabilidad y relevancia de la propuesta curricular para el curso "Recursos Didácticos para la Enseñanza del Inglés" dentro del entorno universitario para la capacitación del estudiantado, según uno de los objetivos específicos de la presente investigación. 
El FODA se aplicó en un grupo de diez estudiantes de la Carrera de Bachillerato en la Enseñanza del Inglés posterior a haber llevado el curso con los lineamientos de la propuesta curricular. Consecutivamente, se procedió a la ejecución del análisis de resultados mediante una tabulación de datos referentes a las debilidades, fortalezas, oportunidades y amenazas del curso, según el criterio de los sujetos participantes

Respecto a la identificación de fortalezas versus debilidades de la propuesta curricular, los resultados se exponen en la Tabla 1.

Tabla 1: Análisis de la validación de la propuesta curricular: Fortalezas versus debilidades

\begin{tabular}{cl}
\hline $\begin{array}{c}\text { \% de } \\
\text { evaluadores }\end{array}$ & \multicolumn{1}{c}{ Aspectos considerados como fortaleza } \\
\hline & - El curso ofrece una diversidad de recursos pre-tecnológicos y tecnológicos que son útiles para la \\
formación del profesorado para la práctica educativa. \\
- El curso ofrece una especial diversidad de recursos que conlleva al manejo del las TIC en la práctica \\
educativa (Román (2006). \\
- El programa ofrece una diversidad de opciones metodológicas para la comprensión de contenidos \\
(Román (2006). \\
- La estructura de los contenidos desarrolla la indagación, la creatividad y el autoaprendizaje a \\
partir de la construcción del conocimiento (Román (2006). \\
- Los contenidos que se presentan son relevantes y actualizados (Román (2006). \\
- El interés de los contenidos del curso, desde un punto de vista teórico, son aplicables para la \\
enseñanza del inglés. \\
- Los contenidos del curso son de carácter práctico para la enseñanza del inglés. \\
- Los ejes temáticos que aborda el curso se califican como innovadores. \\
- Los ejes temáticos del curso buscan potenciar habilidades que generen nuevas ideas o productos \\
mediante la elaboración, creación e innovación. \\
- La metodología del curso motiva y atrae al público usuario a seguir utilizando los recursos \\
didácticos aplicados. \\
- Los ejercicios de tipo formativo que ofrece el curso son útiles para la comprensión y desarrollo de \\
los contenidos (Román (2006). \\
- La inclusión de la TIC ofrece al curso un gran potencial de implementar un entorno de aprendizaje \\
totalmente virtual. \\
• El carácter didáctico-pedagógico de los contenidos es conveniente para la enseñanza del inglés. \\
\hline
\end{tabular}

- El curso busca potenciar el enfoque comunicativo mediante una plena exposición al uso del idioma inglés y una variedad de oportunidades para utilizarlo en diversos contextos orales o $90 \%$ escritos.

- El volumen de la información presentada en el curso apropiado según los contenidos (Román (2006).

- Los diferentes recursos que se ofrecen facilitan la comprensión de los ejes temáticos.

$80 \%$

- El curso permite la utilización de diferentes recursos aptos para enfocar las cuatro macrohabilidades del habla inglesa, específicamente escucha, oralidad, lecto-comprensión y escritura.

Nota: Elaboración con datos propios y datos a partir de Román (2006). 
doi: http://dx.doi.org/10.15359/ree.23-3.11

URL: http://www.una.ac.cr/educare

CORREO: educare@una.cr

Como resultado, se observa que una serie de fortalezas predominan sobre las capacidades de aplicación y características propias del curso; ninguna persona participante encontró alguna posible debilidad. Por ello, se determina que la propuesta curricular efectivamente es aplicable de forma teórico-práctica dentro de la conceptualización, estructuración diseño e implementación de las TIC para la capacitación del estudiantado como futura persona docente. Los resultados reforzaron la identificación de capacidades especiales de aplicación con las que cuenta el curso respecto a la conceptualización, estructuración, diseño e implementación de recursos (tanto pre-tecnológicos como tecnológicos).

De igual forma, en la Tabla 2 se logra apreciar el análisis FODA que permitió identificar las oportunidades versus amenazas de la propuesta curricular según el entorno.

Tabla 2: Análisis de la validación de la propuesta curricular: Oportunidades versus amenazas

$\%$ de evaluadores

$100 \%$
Aspectos considerados como oportunidades

- Las TIC cambian las formas de aprendizaje y surgen como una nueva estrategia para ofrecer mayores oportunidades y acercamiento al conocimiento (Álvarez y Zorrilla, 2008).

- Las TIC representan nuevos escenarios de enseñanza y aprendizaje (Álvarez y Zorrilla, 2008).

- Las universidades buscar ofertar más cursos bimodales y virtuales, para la formación de docentes (Álvarez y Zorrilla, 2008).

- En la enseñanza del inglés, las TIC facilitan una gama de canales sensoriales para representar todos los aspectos lingüísticos que están involucrados en el idioma.

- El paradigma docente favorece la introducción de las TIC en la carrera de inglés para la formación de futuro profesorado.

90\% - El volumen de tecnologías de la información disponibles actualmente es mayor; las tecnologías nacen, avanzan y caducan muy rápidamente (Olivar y Daza, 2007).

- La introducción de internet y la distribución de la información impacta los escenarios educativos educación (Álvarez y Zorrilla, 2008).

- La integración de las TIC en la educación superior modifica los procesos de enseñanza en cuanto a metodologías, modalidades sistemas de comunicación (Álvarez y Zorrilla, 2008).

- Emergen tendencias a implementar enfoques dirigidos a la enseñanza-aprendizaje de un idioma mediante la utilización de las tecnologías en computación.

- En su mayoría, existe en la universidad disponibilidad tecnológica, accesibilidad y usabilidad / navegabilidad de tal forma que se garantice el correcto funcionamiento y desarrollo para los cursos de formación docente.

80\% - Basado en la influencia de las TIC, el rol del profesorado empieza a cambiar notablemente, lo que implica, que deje de ser un transmisor de la información y pasa proponer situaciones de aprendizaje y recursos tomando en cuenta condiciones cognitivas y estilos de aprendizaje (Viñals y Cuenca, 2016).

$70 \%$ - En su mayoría, el profesorado de la enseñanza del inglés posee diversas competencias relacionadas con las TIC en cuanto a: metodologías para el abordaje de contenidos; para selección, diseño e implementación de espacios y recursos educativos (Valencia-Molina et al., 2016).

Nota: Elaboración propia. 
Según los resultados obtenidos respecto a las oportunidades del curso frente a las posibles amenazas implican la identificación de factores provenientes del entorno que dan un carácter significativo a la implementación de las TIC en la propuesta curricular. Se observó un amplio margen en cuanto a las oportunidades generadas; ninguno de los sujetos participantes anotó alguna posible amenaza. Por lo tanto, la propuesta curricular efectivamente se destaca por implementar acertadamente las TIC como elemento significativo y relevante en la capacitación del estudiantado como futura persona docente.

En general, la propuesta curricular cuenta con una serie de fortalezas y oportunidades de cara a la implementación de las TIC como herramienta didáctica significativa en el proceso de enseñanza-aprendizaje del idioma inglés. Sin embargo, para disminuir cualquier eventual debilidad y amenaza, es determinante considerar la necesidad de establecer una planeación estratégica curricular en cuanto a la implementación de las TIC en los procesos de formación docente; desarrollar una capacitación docente continua y eficiente en cuanto al manejo de las TIC en procesos formativos y puntualizar en las competencias concernientes a los nuevos roles del profesorado de cara a la era de la información.

\section{Evaluación del multimedia}

Por otra parte, para la evaluación del multimedia se utilizó como instrumento de recolección de datos el cuestionario, este se utilizó con el fin de facilitar la recogida de información y poder cualificar resultados. Dicha técnica permitió recoger insumos sobre dos grandes dimensiones: los aspectos técnicos y estéticos propios del multimedio y la calidad pedagógica de los contenidos y de los materiales que este ofrece.

El cuestionario referente a aspectos técnicos y estéticos fue evaluado por cinco personas expertas de amplia experiencia en del área de artes gráficas. Por su parte, la calidad pedagógica de los contenidos fue evaluada mediante cinco docentes de la enseñanza del inglés en educación superior. En ambos cuestionarios, los grupos evaluadores cualificaron diversas características y elementos propios de la función del multimedio según su especialidad; posteriormente, se procedió a tabular los datos obtenidos con el fin realizar mejoras al producto.

\section{Aspectos técnicos y estéticos}

A partir de la evaluación de los aspectos técnicos y estéticos, el multimedia obtuvo mayormente calificaciones buenas (la máxima calificación en el instrumento) en los siguientes aspectos:

- la calidad del multimedia;

- la utilización del audio;

- la utilización de imágenes (nitidez, acople a la línea gráfica);

- el diseño gráfico; 
doi: http://dx.doi.org/10.15359/ree.23-3.11

URL: http://www.una.ac.cr/educare

CORREO: educare@una.cr

- el diseño gráfico en cuanto tipología;

- el manejo del color;

- la calidad y fluidez de las animaciones;

- el funcionamiento del multimedio;

- el tiempo de acceso a las diferentes partes del programa;

- la presentación gráfica de la información en cada módulo;

- la claridad para identificar los enlaces en la navegación;

- el funcionamiento de todos los enlaces;

- la navegación en el multimedio;

- la estética del multimedio;

- el funcionamiento técnico del multimedio;

- elfuncionamientodel multimedioen las plataformas PC/MACysus respectivos navegadores.

Sin embargo, el multimedio sí recibió observaciones y recomendaciones puntuales respecto a:

- la calidad del material respecto al diseño gráfico;

- el diseño gráfico en cuanto a tamaño y tipo de letra;

- el manejo del color;

- la presentación gráfica en cada módulo.

Por tanto, se procedió a realizar las mejoras necesarias para cumplir con los objetivos propuestos. De esta forma, para con el manejo del texto, algunos de ellos se consideraron cuantiosos, por lo que, se fragmentaron por párrafos o ideas por línea para evitar generar seguidillas de texto que puedan comprometer la lectura del conjunto. Se enfocó en mantener el puntaje e interlineado a lo largo de todo el multimedia, ya que dicha consistencia optimiza la relación del usuario con la interfaz. Además, se trabajó en jugar más con la variedad del color para generar mayor dinamismo. De esta forma, se agregó color a los títulos grandes y botones seleccionados. Siguiendo parámetros de usabilidad y navegación recomendados por Nielsen (1999) y Morville y Rosenfeld (2006) el identificador del sitio en el mundo occidental debería de estar ubicado en la esquina superior izquierda de la pantalla por el patrón de lectura de izquierda a derecha y de arriba para abajo. Respondiendo a patrones de búsqueda y ubicación por parte del usuario evitando el consumo de tiempo en aprender algo que es intuitivo en la presentación de contenidos para medios digitales. Por tal motivo, se procedió a cambiar el identificador a la esquina superior izquierda del multimedio. Se efectuaron también cambios para maximizar la adecuación del material, de forma que los elementos técnicos y estéticos del multimedio hagan de este un medio apropiado para los propósitos informativos que persigue. Es decir, se obtuvo un balance en los elementos para que el multimedio se adecue a las necesidades como medio informativo efectivo complementario a la propuesta curricular. 
Para los evaluadores, el multimedia se considera como un recurso por medio del cual se puede aprender o informarse de manera muy sintética y directa, el cual es el punto más fuerte del material.

\section{Pertinencia pedagógica de los contenidos y de los materiales}

Con relación a la calidad pedagógica de los contenidos, el multimedio obtuvo una calificación de Siempre en la mayoría de los casos, respecto a:

- la eficacia del módulo introductorio, el usuario identifica el objetivo a alcanzar;

- la existencia de una conexión del multimedio con las necesidades del público meta para el que está diseñado;

- la documentación y los recursos ofrecidos son claramente informativos;

- el formato es claro y coherente de pantalla a pantalla;

- los enlaces externos hacen un uso excelente de Internet y proporcionan información significativa que ayudará al usuario a extender el aprendizaje;

- la información se redacta en forma lógica y clara y se vincula con el multimedia, contiene textos correctamente estructurados;

- no presenta errores gramaticales que dificulten la comprensión;

- la utilización del idioma es apropiada e incluye cadenas léxicas y vocabulario pertinente según el público meta al cual se dirige;

- proporciona herramientas innovadoras y aplicables en la enseñanza del inglés.

Sin embargo, el multimedia recibió recomendaciones y observaciones específicas en cuanto a:

- una conexión significativa entre la información y los recursos que presenta el multimedio;

- la síntesis de diversas fuentes de información y mayor exploración más sobre los datos dados;

- los caracteres de texto utilizados;

- el formato para pasar de una pantalla a otra;

- mínimos errores de ortografía y puntuación.

Al respecto, se procedió a incluir recursos adicionales complementarios a cada herramienta tecnológica, como lo son tutoriales y muestras de trabajos realizados, con el fin de explotar aún más la calidad informativa del multimedio. De igual forma, se trabajó con el sistema de navegación entre pantallas y los caracteres de texto con el fin de lograr mayor claridad y afinidad. Por último, se realizaron cambios con el objetivo de hacer del multimedia un medio pertinente para informar y proporcionar al personal docente nuevas herramientas pedagógicas de aprendizaje significativo integrando las TIC en la enseñanza del inglés. 
doi: http://dx.doi.org/10.15359/ree.23-3.11

URL: http://www.una.ac.cr/educare

CORREO: educare@una.cr

Por otra parte, el multimedio fue considerado como una excelente herramienta didáctica para la incorporación de las TIC a un campo tan necesario como lo es la enseñanza de un idioma extranjero. Posterior a la navegación en el multimedio, se plasma la idea de que la implementación de las TIC es factible tanto desde la perspectiva económica, como para el usuario con poca experiencia en el tema.

\section{Conclusiones y recomendaciones}

\section{Conclusiones}

Dentro de la sociedad del conocimiento, las TIC emergen no solo como un medio de soporte más en el ámbito de la educación, sino también, como un factor de cambio para las bases y principios de los procesos de enseñanza y de aprendizaje. Al respecto, se visualizan nuevas necesidades educativas propias de las constantes transformaciones y exigencias actuales. El mundo tecnológico avanza rápidamente, modificándose de forma continua e interminable. Como se ha evidenciado a través del estudio, la creciente evolución de estos medios afecta directamente a los jóvenes y su proceso de socialización y aprendizaje.

Se logra efectivamente el diseño de una propuesta curricular para el curso "Recursos Didácticos para la Enseñanza del inglés" basado en la conceptualización, estructuración, diseño e implementación de las TIC. Dichas tecnologías se asocian a una transformación en la educación, entre ellas:

a) Potencian el desarrollo de las habilidades del pensamiento superior.

b) El aprendizaje centrado en el alumnado apunta hacia una construcción activa del conocimiento; en la cual, la creación, innovación, y por tanto, la colaboración marcan el desarrollo de nuevas competencias de cara a la sociedad de la información.

c) Expanden el contextoáulico, brindan mayor acceso a recursos actualizados provenientes de áreas nativo-hablantes del idioma y mediante una gran disposición de herramientas. Este aspecto ofrece no solo al estudiante, sino también al docente, una serie de recursos auténticos y significativos que logran una mayor afluencia comunicativa en el idioma inglés.

d) La utilización de videos y multimedia que combine texto, sonido, color y animaciones brindan mayor innovación y autenticidad en el proceso de aprendizaje.

e) Actividades interactivas, como el uso del podcast, logran la creación y combinación de conversaciones, música o discursos que competen al estudiante a escuchar y producir el idioma de una forma más auténtica. 
Así mismo, es imprescindible apuntar hacia una innovación en cuanto a diseño y desarrollo de diversos factores, entre los cuales se encuentran: formatos de comunicación sincrónicos y asincrónicos; instrumentos y procesos de evaluación hacia el desempeño del estudiantado dentro y fuera del aula; y por último, un replanteamiento de los roles del profesorado y del alumnado en la acción educativa.

Sin embargo, aún cuando la implementación de las TIC en la presente propuesta curricular marca un cambio en cuanto a pedagogía e innovación en la educación, es imprescindible que exista una articulación fundamentada entre los recursos didácticos pre-tecnológicos y tecnológicos para la enseñanza del inglés. De esta forma, posterior a su evaluación, dicha propuesta se distingue como aplicable y relevante para el curso "Recursos Didácticos para la Enseñanza del Inglés" dentro del entorno universitario para la capacitación del estudiantado en la enseñanza del inglés.

Por último, es importante señalar el desempeño docente para impartir el curso en un entorno bimodal, tomando en cuenta su nuevo rol como facilitador de la información. Para ello, la producción del material multimedia para la implementación de recursos didácticos para la enseñanza del inglés basados en las TIC representa un recurso complementario acorde con la propuesta curricular. El multimedia cumple con su función de sistema de distribución de información al proporcionar al personal docente que imparta el curso nuevas herramientas pedagógicas de aprendizaje significativo integrando la tecnología con la metodología aplicada en dicha propuesta. La adecuación y pertinencia del diseño del material promueve modelos mentales para el aprendizaje significativo, mediante la autonomía, la participación activa, y la retención y aplicación del conocimiento.

El multimedio no representa únicamente un medio de innovación para los recursos didácticos utilizados en el aprendizaje del idioma inglés. El material ofrece una propuesta para cambiar y adaptar metodologías enfocadas a entornos más auténticos, interactivos y variados según los diversos estilos de aprendizaje.

\section{Recomendaciones}

Posterior a la validación de la propuesta curricular y el material multimedia, se incluye una serie de recomendaciones a los diversos sujetos concernientes al alcance de la propuesta curricular.

a) Al Centro de Investigación y Docencia en Educación, División de Educología:

- El presente diseño curricular con un enfoque bimodal representa una propuesta renovada para el curso "Recursos Didácticos para la Enseñanza del Inglés". Su implementación permite una formación profesional acorde con la sociedad de la información, caracterizada por los grandes avances tecnológicos. 
doi: http://dx.doi.org/10.15359/ree.23-3.11

URL: http://www.una.ac.cr/educare

CORREO: educare@una.cr

- Establecer un perfil docente para impartir el curso bajo un enfoque bimodal, que se muestre capacitado en el manejo e implementación de las TIC en entornos educativos.

- Debido a la rápida evolución de las TIC, los contenidos, recursos y actividades que se incluyen en la presente propuesta curricular deben pasar por un continuo proceso de revisión y actualización. De igual forma, pese a que el multimedio informativo corresponde a herramientas de carácter de innovación, es importante desarrollar proyectos de actualización dentro de sus contenidos para no perder su objetivo.

- Para lograr una exitosa implementación de la propuesta curricular para el curso "Recursos Didácticos para la Enseñanza del Inglés" es necesario llevar a cabo una continua capacitación docente en el manejo e implementación de las TIC tanto en su contenido, como en lo pedagógico, técnico y social. El multimedio aportado mediante el presente estudio constituye un elemento informativo; por tanto, es importante desarrollar capacitaciones formativas para cada herramienta web.

- El éxito de la presente propuesta curricular depende del acceso por parte de docentes y estudiantes a un laboratorio de informática para las sesiones presenciales. El espacio debe estar proveído con equipo de hardware moderno que cumpla con los requerimientos para lograr la implementación de las diversas herramientas tecnológicas; al respecto se hace mención especial a la adquisición de audífonos con micrófono incorporado. De igual forma, es necesario un equipo de software que se mantenga actualizado de manera que se dé acceso libre y navegación de forma óptima en las plataformas requeridas.

- Es importante integrar la tecnología a lo largo de todo el programa de formación docente. La limitación de experiencias relacionadas con tecnologías en un único curso no ayuda al estudiantado a ser capaz de hacer un uso integral de la tecnología desde un punto de vista pedagógico.

b) Al personal docente de la Carrera de Bachillerato en la Enseñanza del Inglés:

- Las TIC ofrecen una gran diversidad de herramientas aplicables para la enseñanza del inglés debido a que permiten focalizar las diferentes macro-habilidades. Sin embargo, debido a la rápida evolución de dichas tecnologías es indispensable mantener una continua formación que instruya sobre las competencias necesarias en el uso e implementación de éstas en la práctica educativa.

- De igual forma, es parte de la responsabilidad del personal docente planificar y dirigir las condiciones esenciales para una inclusión efectiva del las TIC en la formación de profesorado.

- No se debe perder la perspectiva sobre el hecho de que el futuro personal debe formarse y experimentar dentro de entornos educativos que hagan un uso innovador y pertinente de la tecnología. 
- Es importante recalcar que la implementación de las TIC en los procesos formativos no debe sustituir los recursos tradicionales; por el contrario, dichas tecnologías deben ser utilizadas con el fin de complementar y optimizar los procesos de formación docente.

- Por último, al asumir un curso de carácter bimodal, el profesorado no debe evitar caer en el error de pensar que las estructuras de los contenidos, los métodos, los recursos didácticos, los medios de comunicación o las actividades que se utilizan en la modalidad presencial son las mismas que se pueden utilizar en cursos bimodales. Consecuentemente, parte del reto es que el personal docente debe aprender nuevamente a ser personal docente universitario; y el aprendizaje del uso de las tecnologías de la información y las comunicaciones es tan solo una parte del desafío que plantea el aprendizaje en forma bimodal.

b) A futuras investigaciones:

- Con base en la interactividad, flexibilidad y accesibilidad que posibilita la implementación de las TIC en el curso con un enfoque bimodal, se genera entonces una oportunidad de investigación sobre su adecuación en un entorno virtual; lo cual implicaría que la mayoría, sino toda, la instrucción y evaluación se lograrían vía recursos accesibles en la web. Esto podría resolver diversos problemas educativos presentes hoy día, desde el acceso/aislamiento geográfico, ahorro de tiempo y dinero, hasta incluso el satisfacer las demandas presentes en la era de la información.

\section{Referencias}

Álvarez, E. y Zorrilla, M. (2008). Orientaciones en el diseño y evaluación de un curso virtual para la enseñanza de aplicaciones informáticas. IEEE-RITA, 3(2), 61-70. Recuperdo de http://rita. det.uvigo.es/200811/uploads/IEEE-RITA.2008.V3.N2.A1.pdf

Brown, H. D. (2001). Teaching by principles. An interactive approach to language pedagogy. New York: Addison Weasley Longman.

Cabero, J. (2001). Tecnología educativa: Diseño y utilización de medios en la enseñanza. Barcelona: Paidós.

Cabero, J. (2007). Tecnología educativa: Su evolución histórica y su conceptualización. En J. Cabero (Coord.), Tecnologíaeducativa (pp. 13-28). España:McGraw-Hill. Recuperadode http://novella. mhhe.com/sites/dl/free/8448156137/471653/Capitulo Muestra Cabero 8448156137.pdf 
doi: http://dx.doi.org/10.15359/ree.23-3.11

URL: http://www.una.ac.cr/educare

CORREO: educare@una.cr

Cabero, J. (2008). Innovación en la formación y desarrollo profesional docente. En J. Salinas (Coord.), Innovación educativa y uso de las TIC (pp. 83-99). Sevilla: Universidad Internacional de Andalucía. Recuperado de http://dspace.unia.es/bitstream/10334/130/1/004tic cabero.pdf

Cabero-Almenara, J. y Ruiz-Palmero, J. (2017). Las tecnologías de la información y comunicación para la inclusión: Reformulando la brecha digital. IJERI: International Journal of Educational Research and Innovation, 9, 16-30. doi: http://orcid.org/0000-0002-6958-0926

Churches, A. (2009). Taxonomía de Bloom para la era digital. Recuperado de http://www.eduteka. org/TaxonomiaBloomDigital.php

Correa, S., Puerta, A. y Restrepo, B. (1996). Investigación evaluativa. Colombia: ICFES. Recuperado de http://en.calameo.com/read/0000914544f02278de394

Dudeney, G. y Hockly, N. (2007). How to... teach English with technology. England: Pearson Education. Doi: https://doi.org/10.1093/elt/ccn045

Morville, P. y Rosenfeld, L. (2006). Information architecture for the world wide web:Designing largescale web Sites. California: O'Reilly Media.

Nielsen, J. (1999). Designing web usability. California: PeachPit.

Nunan, D. (2003). Methodology. En D. Nunan (Ed.), Practical English language teaching (pp. 3-22). New York: Mc Graw Hill Contemporary.

Olivar, A. J y Daza, A. (2007). Las tecnologías de la información y comunicación (TIC) y su impacto en la educación del siglo XXI. Negotium, 3(7), 21-46. Recuperado de https://www.redalyc. org/html/782/78230703/

Román, P. (2006). Las presentaciones de los alumnos. En J. Cabero y P. Román (Coords.), E-actividades. Un referente básico para la formación en Internet (pp. 113-138). Sevilla: Editorial MAD. Recuperado de https://es.scribd.com/document/287800272/Cabero-EActividades-Un-Referente-Basico-Para-La-Formacion-en-Internet

Serrano, J. M. y Pons, R. M. (2011). El constructivismo hoy: Enfoques constructivistas en educación. Revista Electrónica de Investigación Educativa, 13(1), 1-27. Recuperado de http://www. scielo.org.mx/pdf/redie/v13n1/v13n1a1.pdf

Ulate, G. (2009). Implementación de curso Recursos Didácticos para el Aprendizaje bajo un enfoque bimodal para las nueve áreas de la especialidad a las cuales se dirige el curso (Informe Núm. I, Código 010903). Heredia: Universidad Nacional. 
Universidad Nacional, Centro de Investigación y Docencia en Educación, División de Educología. (2005). Programa del curso: Recursos didácticos para el aprendizaje de inglés (Código DEY468, Programa de estudio). Heredia, Costa Rica: Autor.

Universidad Nacional, Centro de Investigación y Docencia en Educación, División de Educología. (2008). Programa del curso: Recursos didácticos para el aprendizaje (bimodal, Código DEX 320,). Heredia, Costa Rica: Autor.

Valencia-Molina, T., Serna-Collazos, A., Ochoa-Angrino, S., Caicedo-Tamayo, A. M., MontesGonzález, J. A. y Chávez-Vescance, J. D. (2016). Competencias y estándares TIC desde la dimensión pedagógica: Una perspectiva desde los niveles de apropiación de las TIC en la práctica educativa docente. Cali: Pontificia Universidad Javeriana. Recuperado de http:// www.unesco.org/new/fileadmin/MULTIMEDIA/FIELD/Santiago/pdf/Competenciasestandares-TIC.pdf

Viñals, A. y Cuenca, J. (2016). El rol del docente en la era digital. Revista Interuniversitaria de Formación del Profesorado, 30(2), 103-114. Recuperado de https://dialnet.unirioja.es/ servlet/articulo?codigo $=5670199$ 